Originalveröffentlichung in: Filip Coppens, Jiři Janák, Hana Vymazalová (Hg.), Royal versus divine authority. Acquisition, legitimization and renewal of power; 7. Symposion zur ägyptischen Königsideologie / 7th Symposium on Egyptian Royal Ideology; Prague, June 26-28, 2013, (Königtum, Staat und Gesellschaft früher Hochkulturen 4,4), Wiesbaden 2015, S. 49-61

\title{
Die Erneuerung der königlichen/göttlichen Macht und das Lebenshaus von Ra-sehet (Fayum)
}

\author{
Horst Beinlich
}

Das ägyptische „Buch vom Fayum“ läßt sich inhaltlich in zwei Teile teilen, die mit der physikalischen Aufteilung des „Buches vom Fayum“ in der Handschrift „Boulaq/Hood/ Amherst" in zwei Papyrusrollen übereinstimmt.

Im ersten Teil des „Buches vom Fayum“ wird auf der Basis einer Landkartenstruktur die Rolle von zwei Göttern beschrieben. Die Landkarte beinhaltet dabei den Bereich des Bahr Yussuf kurz vor dem Eintritt in das Fayum, seinen Weg durch die Lahun-Schwelle bis etwa nach Krokodilopolis, die Bereiche, die vom Bahr Yussuf mit Wasser versorgt werden, und schließlich den See, der sich in der tiefsten Stelle der Fayum-Depression ausbreitet.

Der Weg des Wassers bzw. des Überschwemmungswassers, das so das Fayum erreicht, wird mit Osiris verbunden. Aus den mythischen Nilquellen bei Elephantine kommend wird das fruchtbare Überschwemmungswasser des Nils (= Osiris) bei El-Lahun (teilweise) ins Fayum abgeleitet und findet getreu der Vorstellung, daß Osiris Herrscher des Westens ist, im westlichen Bereich des Fayumsees seine Ruhestätte.

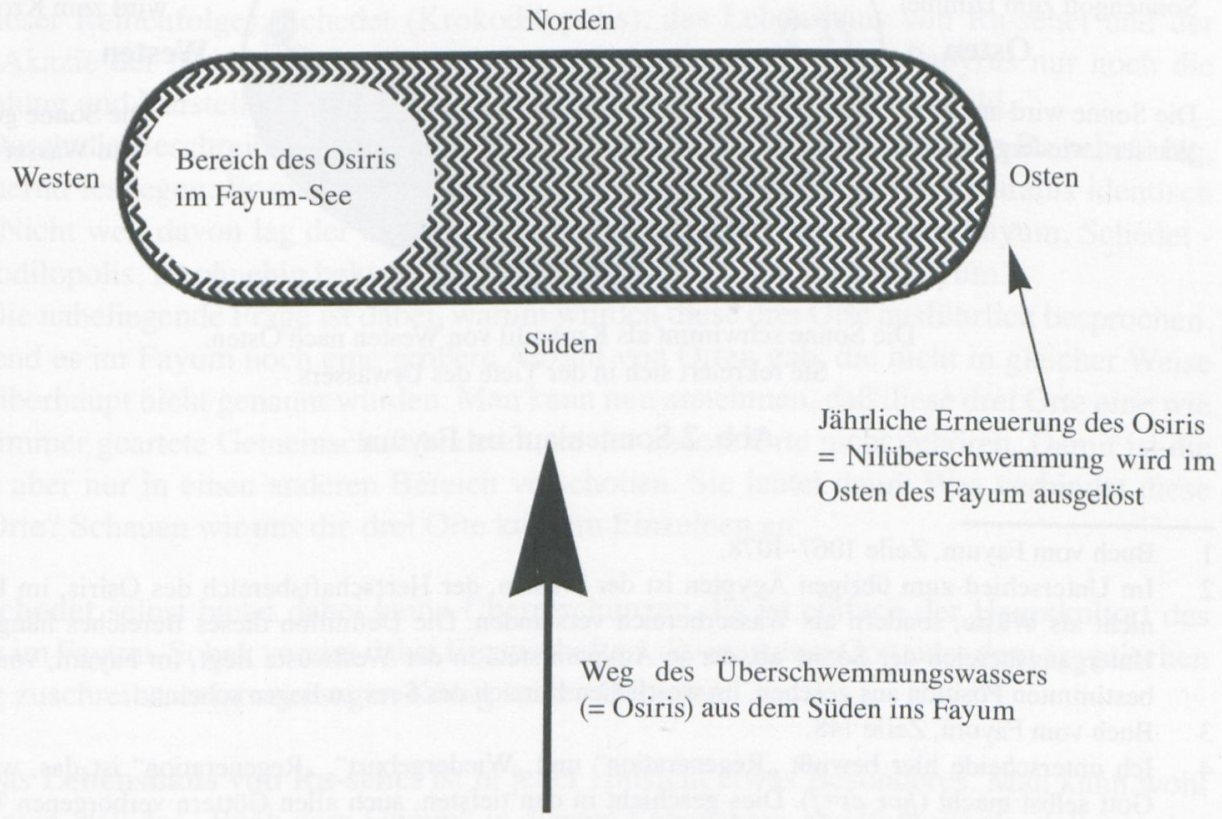

Abb. 1 Osiris im Fayum 
Da die Vorstellung von Osiris an die jährliche zyklische Erneuerung der Natur gebunden ist, kann auch diese Ruhestätte des Osiris im Fayum nur für ein Jahr ihre Gültigkeit haben. Von hier beginnt Osiris seine Regeneration, indem er auf dem Weg durch das unterirdische Urgewässer (Nun) regeneriert wird und aus den Nilquellen bei Elephantine wieder zutage tritt. Ausgelöst wird diese Regeneration im Lebenshaus von Ra-sehet ${ }^{1}$ im Osten des Fayum. Es ist auffallend, daß Osiris außer im Bereich des Bahr Yussuf nur im Westen des großen Sees des Fayum eine (passive) Rolle spielt. ${ }^{2}$ Man muß dabei selbstverständlich unterscheiden: Osiris als fruchtbares Überschwemmungswasser ist nur partiell mit Osiris, dem Herrscher des Totenreiches gleichzusetzen. Immerhin haben die Verfasser des „Buches vom Fayum“ den See als Ort, ,an dem Osiris ruht und als Ort, an dem die Westlichen begraben sind“ verstanden. ${ }^{3}$

Durch die Sonne wird nicht nur der Ort des Osiris - Ort des Sonnenuntergangs - definiert, auch das übrige Fayum ist in den Lauf der Sonne und seine mythologische Umsetzung eingebunden. Der Fayum-See ist die Urheimat des Sonnengottes. Hier ist er von der Mehetweret, der großen Flut, im Beisein der acht Urgötter geboren worden. Und hier im See des Fayum regeneriert er sich jeden Tag auf's Neue und wird anschließend neu geboren. ${ }^{4}$

Er überquert den Himmel von Ost nach West, taucht am Westufer des Sees in das Wasser ein, nimmt die Gestalt eines großen Krokodils an, wobei er sich regeneriert, und

Leserichtung des „BUCHES vOM FAYUM“ von links (Osten) nach rechts (Westen)

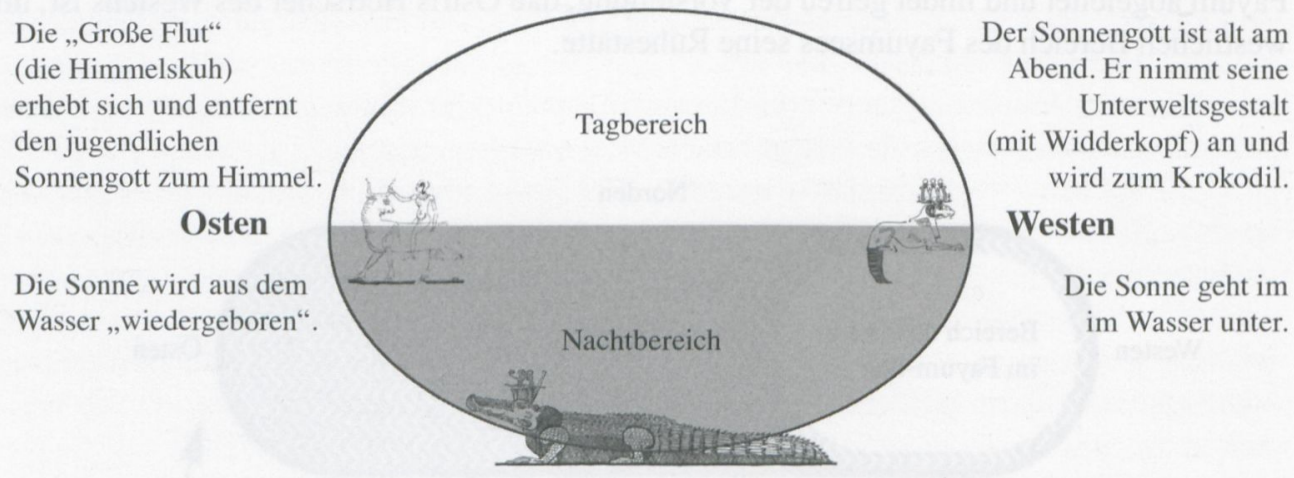

Die Sonne schwimmt als Krokodil von Westen nach Osten.

Sie rekreiert sich in der Tiefe des Urwassers.

Abb. 2 Sonnenlauf im Fayum

1 Buch vom Fayum, Zeile 1067-1078.

2 Im Unterschied zum übrigen Ägypten ist der Westen, der Herrschaftsbereich des Osiris, im Fayum nicht als Wüste, sondern als Wasserbereich verstanden. Die Definition dieses Bereiches hängt vom Untergangsbereich der Sonne ab, der in Ägypten stets in der Westwüste liegt, im Fayum, von einer bestimmten Position aus gesehen, im westlichen Bereich des Sees zu liegen scheint.

3 Buch vom Fayum, Zeile 148.

4 Ich unterscheide hier bewußt „Regeneration“ und „Wiedergeburt“. „Regeneration“ ist das, was der Gott selbst macht ( $h p r \underline{d} s=f$ ). Dies geschieht in den tiefsten, auch allen Göttern verborgenen Tiefen. Die „Wiedergeburt“ ist dann die Folge der „Regeneration“. Dazu braucht der regenerierte Gott eine Muttergottheit. 
schwimmt dann in der Nacht zum Ostufer des Sees. Dort wird er in der Gestalt des Re von der Himmelskuh an den Himmel gehoben (geboren) und beginnt seine Reise von neuem. Die Himmelskuh hat dabei die Funktion derjenigen Götter, die am Ende der Unterweltsbücher den Sonnengott wieder zum Himmel emporheben. Das Ostufer des Fayum-Sees ist deshalb der Ort der Regeneration und Wiedergeburt. Der See des Fayum ist im „Buch vom Fayum“ der Bereich, der den Lauf des Sonnengottes in der Nacht zum Inhalt hat. Die anderen Bereiche des ersten Teils des Papyrus haben mit dem täglichen Sonnenlauf wenig zu tun. Ihre Aufgabe in Bezug auf den Sonnengott ist es darzustellen, wie es denn dazu kam, daß die Sonne ihren Weg in das Fayum genommen hat. Dazu wird auf das Motiv der Verschwörung der Menschen und Götter von Herakleopolis gegen den Sonnengott und die Erhebung des Sonnengottes durch die Himmelskuh an den Himmel zurückgegriffen. Die Entfernung des Sonnengottes an den Himmel wird parallel zur Entfernung des Sonnengottes vom Niltal in das Fayum gesetzt. Sie ist der Beginn einer Weltordnung, die das Fayum als Zentrum sieht. Die Darstellung der großen Himmelsgöttin Mehet-weret gehört durch ihre Texte eindeutig zu der Erzählung von dieser Neuordnung. Die daran anschließende Darstellung des Ostufers des Fayumsees mit den beiden Götterbarken und dem Sonnengott, der zwischen ihnen aus dem See geboren wird, zeigt das Muster der sich täglich wiederholenden Geburt des Sonnengottes. Dabei ist es unerheblich, ob die Schöpfer dieser Darstellung das „erste Mal“ dieser Geburt oder ihre Wiederholung meinten.

Der Sinn der landkartenartigen Darstellung des ersten Teils des „Buches vom Fayum“ ist es, diesen genannten Aspekten des Osiris und des Re einen Rahmen zu geben. Die Götter und Orte am Rande des Zentralgeschehens können mit realen Gegebenheiten des Fayum in Einklang stehen, müssen es aber nicht.

Der zweite Teil des „Buches vom Fayum“ unterscheidet sich deutlich vom ersten Teil. Hier sind drei Orte durch ihre ausführliche Beschreibung besonders hervorgehoben, d.i. (in dieser Reihenfolge): Schedet (Krokodilopolis), das Lebenshaus von Ra-sehet und der Ort „Akazie der Neith“. Außer ihnen haben wir in diesem Teil des Papyrus nur noch die Erzählung und Darstellung von der Gründung des Fayum und das Schlußbild.

Durch die Beschreibung kann man den Ort, an dem das „Lebenshaus von Ra-sehet“ lag, annähernd festlegen. Er müßte bei Karanis gelegen haben oder sogar mit Karanis identisch sein. Nicht weit davon lag der Ort „Akazie der Neith“. Der Hauptort des Fayum, Schedet Krokodilopolis, ist ohnehin bekannt, er lag beim heutigen Medinet el-Fayum.

Die naheliegende Frage ist dabei, warum wurden diese drei Orte ausführlich besprochen, während es im Fayum noch eine größere Anzahl von Orten gab, die nicht in gleicher Weise oder überhaupt nicht genannt wurden. Man kann nun annehmen, daß diese drei Orte eine wie auch immer geartete Gemeinschaft bildeten, zu der andere Orte nicht gehören. Damit ist die Frage aber nur in einen anderen Bereich verschoben. Sie lautet dann: Was verbindet diese drei Orte? Schauen wir uns die drei Orte kurz im Einzelnen an:

Schedet selbst bietet dabei keine Überraschungen. Es ist einfach der Hauptkultort des Sobek im Fayum. Sobek vereint dabei Eigenschaften, die man dem Gott oder dem ägyptischen König zuschreibt, er ist sozusagen König seines Gebietes.

Das Lebenshaus von Ra-sehet ist in jeder Hinsicht etwas Besonderes. Man kann wohl annehmen, daß das „Buch vom Fayum“ in diesem Lebenshaus abgefaßt wurde, daß wir also bei dem, was über das Lebenshaus gesagt wird, Informationen aus erster Hand haben. 


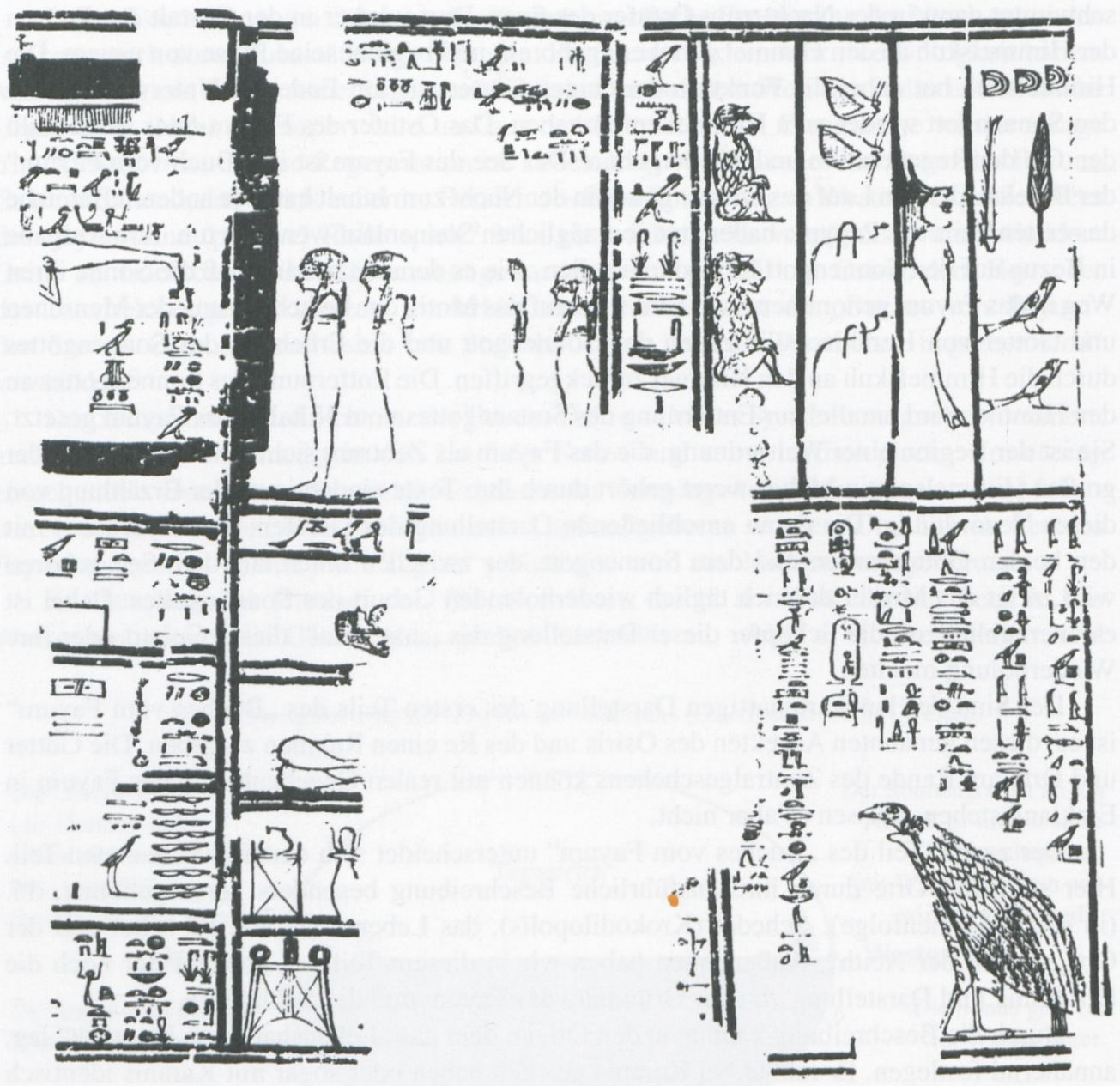

Abb. 3 Das Lebenshaus von Ra-sehet

Im „Buch vom Fayum“ werden im Abschnitt über das „Lebenshaus von Ra-sehet“ zunächst einmal die Baulichkeiten mit ihrer Funktion ausführlich beschrieben bzw. im Bild dargestellt. Danach folgt mit dem kryptographisch geschriebenen Motto des Lebenshauses das Bekenntnis zum Wert uralter Tradition. Darauf folgt ein Text, den die demotischen Parallelen und Erläuterungen als „Schrift (Lied) der Geierin“ bezeichnen. 5

Die aus dem Süden kommt, Nechbet ist es.

Die aus dem Norden kommt, Wadjet ist es.

Die aus dem Westen kommt, Sachmet ist es.

Die aus dem Osten kommt, Neriut ist es. 


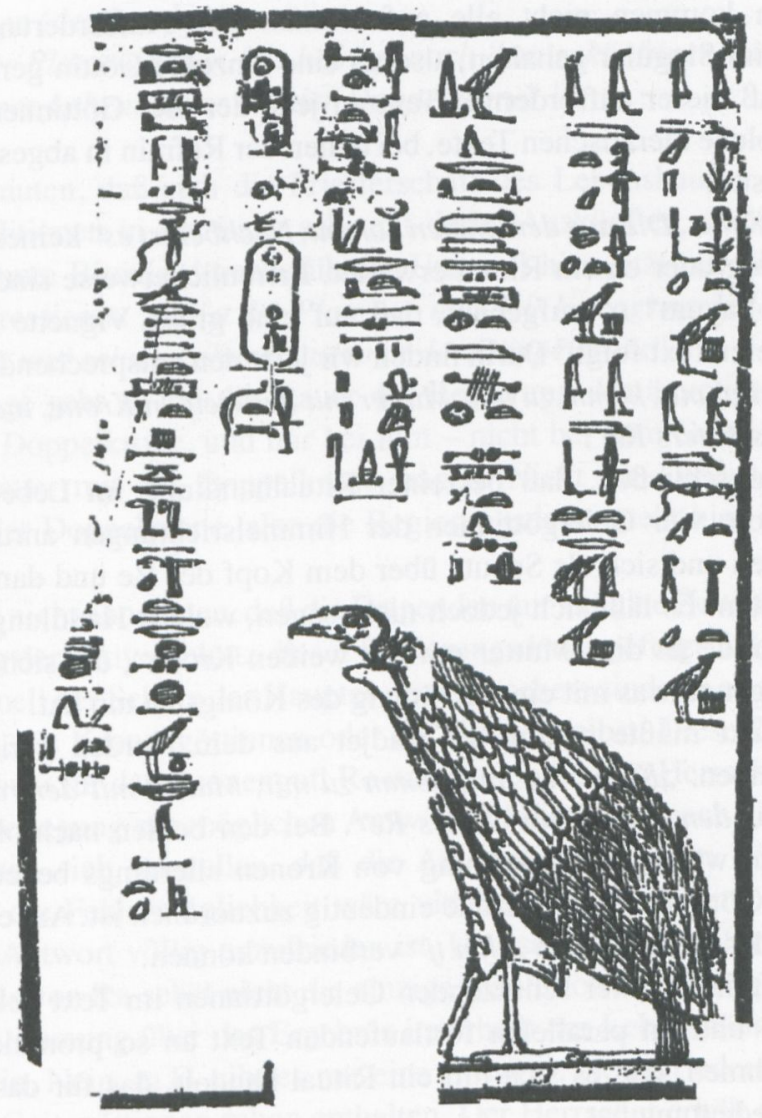

Abb. 4 Das „Lied der Geierin“

Danach folgt:

Möge sie das beschützen, was gegeben ist in das Seeland und nach Mehu, indem sie sich ausbreitet über die Lebenszeit ihres Vaters Re. Möge sie sich geben auf den Kopf des Re-nefer, des Sohnes des Re, Re-nefer, er lebe ewig. Gegeben werde der (fruchtbare) Sand des Wadj-wer (oder: zum Wadj-wer) dieses Sees unter die Füße dieser Göttin.

Re-nefer ist dabei als „rn - Name“ aufzulösen. Damit hätten wir den wichtigsten Bestandteil der Königstitulatur: „Kartuschen-Name (leer), Sohn des Re, Kartuschen-Name (leer)“. Der Text bezieht sich also auf König NN. Die vier Geiergöttinnen kommen also aus den vier Himmelsrichtungen, um den Kopf des Königs zu schützen. Der „Schutz des Kopfes" gehört zu den Aufgaben von Geier-Göttinnen wie Nechbet, die mit Krone und Königskopftuch verbunden wird. ${ }^{6}$

$6 \quad$ Mit der Nennung der vier Himmelsrichtungen wird eine Fokussierung auf einen Punkt erreicht, von dem etwas ausgeht bzw. zu dem etwas hinführt. Dazu gehören etwa das „Schießen von Pfeilen“, das „Aussenden von Botenvögeln“, das „Werfen von Bällen“, das viermalige Umschreiten von etwas usw. 
Die Göttinnen kommen nicht alle auf einmal. Die Aufforderung „Möge sie das beschützen ..." ist im Singular gehalten, also an eine einzelne Göttin gerichtet. Wir können also annehmen, daß dieser auffordernde Text an jede der vier Göttinnen einzeln gerichtet wird. Man kennt solche hieratischen Texte, bei denen der Refrain in abgesetzter Weise neben dem Text plaziert ist.

Nun ist ein Satz wie ,Die aus dem Süden kommt, Nechbet ist es“ keineswegs ein Satz, den man in einem Dialog oder einem Ritual erwartet. Erfreulicherweise sind die Texteinheiten des „Buches vom Fayum“ so aufgebaut, daß auf eine große Vignette zu einem Ort, ein langer, dazu paralleler Text folgt. ${ }^{7}$ Darin finden wir jetzt den entsprechenden Satz mit Isis als Sprecherin: „Komm zu mir, komm zu mir, Mutter mit der weißen Krone, indem sie sich breitet über den Kopf ihres Vaters Re".

Man kann also schließen, daß bei einer Ritualhandlung im Lebenshaus die Göttin Isis nacheinander die vier Geiergöttinnen der Himmelsrichtungen anruft, damit sie zum Lebenshaus kommen und sich als Schutz über dem Kopf des Re und damit über dem Kopf des Königs ausbreiten. Es läßt sich jedoch nicht sagen, welche Handlung damit verbunden ist, ob gar eine Anrede an die „Mutter mit der weißen Krone“, die sicher mit Nechbet zu identifizieren ist, irgendetwas mit einer Krönung des Königs zu tun hat.

Der nächste Text müßte analog an Wadjet aus dem Norden gerichtet werden und folgendermaßen heißen: „Komm zu mir, komm zu mir, Mutter mit der roten Krone, indem sie sich breitet über den Kopf ihres Vaters Re". Bei den beiden nachfolgenden Göttinnen Sachmet und Neriut wäre eine Zuordnung von Kronen allerdings bedeutend schwieriger, weil diesen beiden Göttinnen keine Krone eindeutig zuzuordnen ist. Am ehesten würde man noch Sachmet mit der Doppelkrone ,shm.tj“ verbinden können.

Da das Ritual mit den vier schützenden Geiergöttinnen im Text neben den Vignetten - sogar mit Bild - und im parallelen fortlaufenden Text an so prominenter Stelle zitiert ist, muß man annehmen, daß es sich um ein Ritual handelt, das für das Lebenshaus eine außerordentliche Bedeutung hat.

Wenn man sich fragt, was noch so große Bedeutung für das Lebenshaus hat, daß es von Insidern im Text über das Lebenshaus erwähnt werden muß, dann kommt man sehr schnell zu einer Antwort. Das Lebenshaus ist ein Ort für Orakelfragen an die Götter und zwar für wichtige staatstragende Orakel. (Ich benutze hier das Wort Orakel, obwohl eher eine prophetische Aussage oder ein Horoskop gemeint ist.)

„Es ist der Platz, wo man die Achtheit befragt nach dem zukünftigen Tun der Väterund-Nun (das ist der See im Fayum)“.?

„Es ist der Platz, wo man Geb befragt nach dem zukünftigen Tun seiner Kinder". ${ }^{10}$ „Es ist der Platz des Erweckens (Erwachens) der beiden (Quell)höhlen. Man fragt durch den (oder als) Cheriheb nach den Plänen wegen der Suche nach Osiris, bei diesem Vorbeigehen an Ober- und Unterägypten, an den Tempeln, an den

S. z.B. im LÄ, s.v. Himmelsrichtung. Eine Vierfachnennung von Kronengöttinnen ist m.W. bisher nicht bezeugt. In den "Hymnen an das Diadem der Pharaonen" werden nur drei Kronen und die zugehörigen Göttinnen genannt.

7 Eigentlich ist es wohl so, daß die Vignette folgt, die ganze Einheit Vignette+Text also von rechts nach links verstanden werden muß.

8 Buch vom Fayum, Zeile 1052.

9 Buch vom Fayum, Zeile 1030.

10 Buch vom Fayum, Zeile 1033. 


\section{Niederlassungen (des Landes?) ..."11}

Und „Es ist der Platz, wo man Isis befragt nach dem zukünftigen Tun ihres Sohnes Horus und seiner Lebensdauer mit der Doppelkrone“. ${ }^{12}$

Man darf vermuten, daß sich die Priesterschaft des Lebenshauses als Bewahrer alter Schriften und Traditionen in der Lage sah, mit derlei Auskünften zu dienen. Dazu waren ganz sicher bestimmte Rituale durchzuführen. Uns soll hier jetzt nur die zuletzt genannte Orakelanfrage interessieren: „Es ist der Platz, wo man Isis befragt nach dem zukünftigen Tun ihres Sohnes Horus und seiner Lebensdauer mit der Doppelkrone".

Bei dieser Frage geht es um die Dauer der Regierung des ägyptischen Königs. Er ist der Horus mit der Doppelkrone, und nur bei ihm - nicht bei dem Gott Horus - würde man nach der Lebensdauer mit der Doppelkrone fragen. Nicht die Lebensdauer, sondern die Lebensdauer mit der Doppelkrone, also die Regierungsdauer steht hier im Mittelpunkt des Interesses.

Man darf wohl nicht annehmen, daß die Fragenden nur auf eine Zeitangabe hofften. Nein, hier ist gleichzeitig der aktive Schutz dieser Regierungsdauer gefragt. Isis ruft die Göttinnen herbei, die traditionell der Schutz des Hauptes des Herrschers sind, sei es als Schutzgöttinnen des Kopfbereiches, als Kronengöttinnen oder als Kronen selbst. Diese Göttinnen bewirken einen Rundum-Schutz für den Sonnengott Re und analog für den Herrscher Ägyptens.

Es sei eine Überlegung zur möglichen Antwort auf die Frage nach der Regierungsdauer erlaubt. Könnte man sich vorstellen, daß die Antwort etwa lauten könnte „noch sieben Jahre"? Nicht nur die Fehlermöglichkeit wäre viel zu groß. Es könnte auch sein, daß ein Herrscher mit der Antwort völlig unzufrieden ist. Und schließlich muß man damit rechnen, daß das Lebenshaus von Ra-sehet nicht die einzige Institution ist, von der man eine Antwort erwartet. Eine Abstimmung über das Ergebnis innerhalb der Lebenshäuser Ägyptens dürfte illegal gewesen sein. Nein, m.E. gibt es nur eine Antwort, die zufriedenstellt, und sie ist in der Anrede an die Geiergöttinnen schon enthalten. Der Herrscher erhält die „Lebenszeit des Re“ oder es wird eine andere unbestimmte Angabe gewählt. Im „Buch vom Fayum“ wird das so ausgedrückt: „Es ist Horus, gefestigt wird für ihn seine Lebenszeit auf der Erde in Herzensfreude, ewig." 13

Orakelauskünfte über Dinge zu bekommen, die eine Privatperson betreffen, dürfte nicht all zu schwer gewesen sein. Mit den hier zitierten Orakelanfragen befinden wir uns aber auf einem ganz anderen Niveau. Hier kann nicht jeder zu jedem Augenblick fragen, wie lange der Herrscher noch regieren wird. Für eine solche Frage muß man legitimiert sein und - es muß ein Anlaß dafür gegeben sein.

Die Antwort auf die Orakelfrage wird im „Buch vom Fayum“ gegen Ende des Textes über das Lebenshaus gegeben: „Schutz wird ausgeübt für Horus. Er ist der lebende Ba, trefflich an Leben in Herakleopolis, der lebende Ba in seinem Körper. Kräftig ist Horus mit seinen beiden Armen, mit seiner Kraft, indem er das Land schützt mit seinen Waffen. Er hat sie ergriffen, um seine Feinde zu vertreiben aus den Kampfplätzen. Kräftig ist der König von Ober- und Unterägypten Horus, Pharao (oder: Autokrator), er lebe, sei heil und gesund - in seiner Lebenszeit." ${ }^{\text {"14 }}$

11 Buch vom Fayum, Zeile 1070 bis 1074.

12 Buch vom Fayum, Zeile 1033 bis 1036.

13 Buch vom Fayum, Zeile 1004 bis 1007.

14 Buch vom Fayum, Zeile 1091 bis 1100. 
Bei der Frage nach der Legitimation der Fragesteller kann man davon ausgehen, daß es sich stets um die Priesterschaft des „Lebenshauses von Ra-sehet“ bzw. um die des SobekTempels von Schedet gehandelt hat. Beim Anlaß der Anfrage kann man sich ein konkretes Ereignis vorstellen oder einen Zeitpunkt, zu dem eine solche Frage gehört. Hätten wir ein konkretes Ereignis, so wäre dieser Vorgang sicherlich nicht im „Buch vom Fayum“ festgehalten worden. Dieser Text ist nicht für ein Jahr geschrieben, sondern schreibt die Wiederholung sozusagen für alle Ewigkeit fest. Der einzige Anlaß für eine solche Anfrage ist m.E. die Erneuerung der königlichen Macht, die dann aber nicht als Wiederholung = Erneuerung des realen Amtsantrittes angesehen werden darf, sondern zu einem festen Termin innerhalb des Jahres. Da nun die Daten, die für die Rituale im Ort „Akazie der Neith“ mit „23. Thot“ auf den Jahresanfang deuten, vermute ich, daß die Erneuerung der königlichen Macht in den Rahmen der jährlichen Erneuerung am Jahresanfangs eingebunden wird.

Das Problem des Jahresanfanges liegt sicher auch in der Frage, ob wir das damit verbundene Datum 1. Thot als ein Datum des Naturjahres oder des bürgerlichen Verwaltungsjahres verstehen. Gehen wir davon aus, daß der 1. Thot dem 29. August des julianischen Kalenders und dem 18. Juli des gregorianischen Kalenders entspricht. Nach der Angabe von Wansleb(en) erscheint die Nilüberschwemmung am 2. August (Gregorianischer Kalender) im Fayum. Dies würde dem 16. Thot entsprechen. Nun ist es unmöglich zu sagen, wie lange sich die Rituale um das Neujahrsfest hingezogen haben. ${ }^{15}$

Wenn wir bisher immer von den Ritualen für den König gesprochen haben, so ist das in gewisser Weise eine Fiktion. Der König der römischen Zeit residierte in Rom, der König der ptolemäischen Zeit prinzipiell in Alexandria. Seine persönliche Teilnahme an Ritualen im Fayum kann ausgeschlossen werden. Das Ritual der Erneuerung scheint sich jedoch nicht nur auf die königliche Kraft zu beziehen. Auch die göttliche Kraft erfährt eine Erneuerung, schließlich ist der König Horus. In den Texten, die wir als Antwort an die Orakelanfrage angesehen haben, wird diese Parallelität deutlich:

\section{„Kräftig ist Horus mit seinen beiden Armen ... Kräftig ist der König von Ober- und Unterägypten Horus, Pharao“}

Wenn man darüber hinaus sieht, daß Horus im Fayum mit Sobek gleichgesetzt ist ${ }^{16}$, wird man schließen können, daß in den Fällen, wenn nach dem Ritual eigentlich der König der Begünstigte ist, eine (Haupt)gottheit, Sobek oder/= Horus an seine Stelle treten kann. Sofern man diese Hypothese akzeptiert, ergeben sich einige weitere Überlegungen.

Vor allem hätte man mit der genannten Hypothese einen Ansatz zum Verständnis des Ortes „Akazie der Neith“.

Bislang mußte man „Akazie der Neith“ verstehen, wie der Ort im „Buch vom Fayum“ beschrieben ist, als ein Heiligtum der Neith, in dem Rituale für den König durchgeführt wurden. Die Frage, warum Neith dort ,ihren Horus beschützt, der sich zwischen ihren

15 Im Ritual der „Confirmation du pouvoir royal au nouvel an“ macht der König zunächst im „Lebenshaus“ halt, zieht dann ins , sh $n$ smn jwe $t^{*}$, bleibt dort während der Epagomenen und der ersten 10 Tage des Neuen Jahres und zieht dann wieder zum Lebenshaus. Man kann aber nicht ausschließen, daß die Orakelanfrage durchgeführt wurde, während der König noch im „sh $n \operatorname{smn} j w^{c} \cdot t^{*}$ war. Es scheint mir aber unwahrscheinlich zu sein, daß die Rituale zum Neuen Jahr und die genannten Rituale zur Orakelanfrage so eng beieinander liegen, ohne etwas miteinander zu tun zu haben.

16 Gleichsetzung von Sobek mit dem kämpferischen Horus: s. BF-Zeile 52, mit dem Horus als Kind: s. BF-Zeile 1141 . 
Schenkeln befindet (BF-Zeile 1141)“ und der in der Vignette als Kind mit Krokodilskopf dargestellt ist, stellte sich zunächst gar nicht. Allerdings ist es schon auffallend, daß es hier einen Ort gibt, an dem ein Gott seine Kindheit im Schutz seiner Mutter verbringt und daß dieser Ort dann auch eine Insel ist und von Schilf und Rohr bewachsen. Die Parallele zu Chemmis drängt sich geradezu auf.
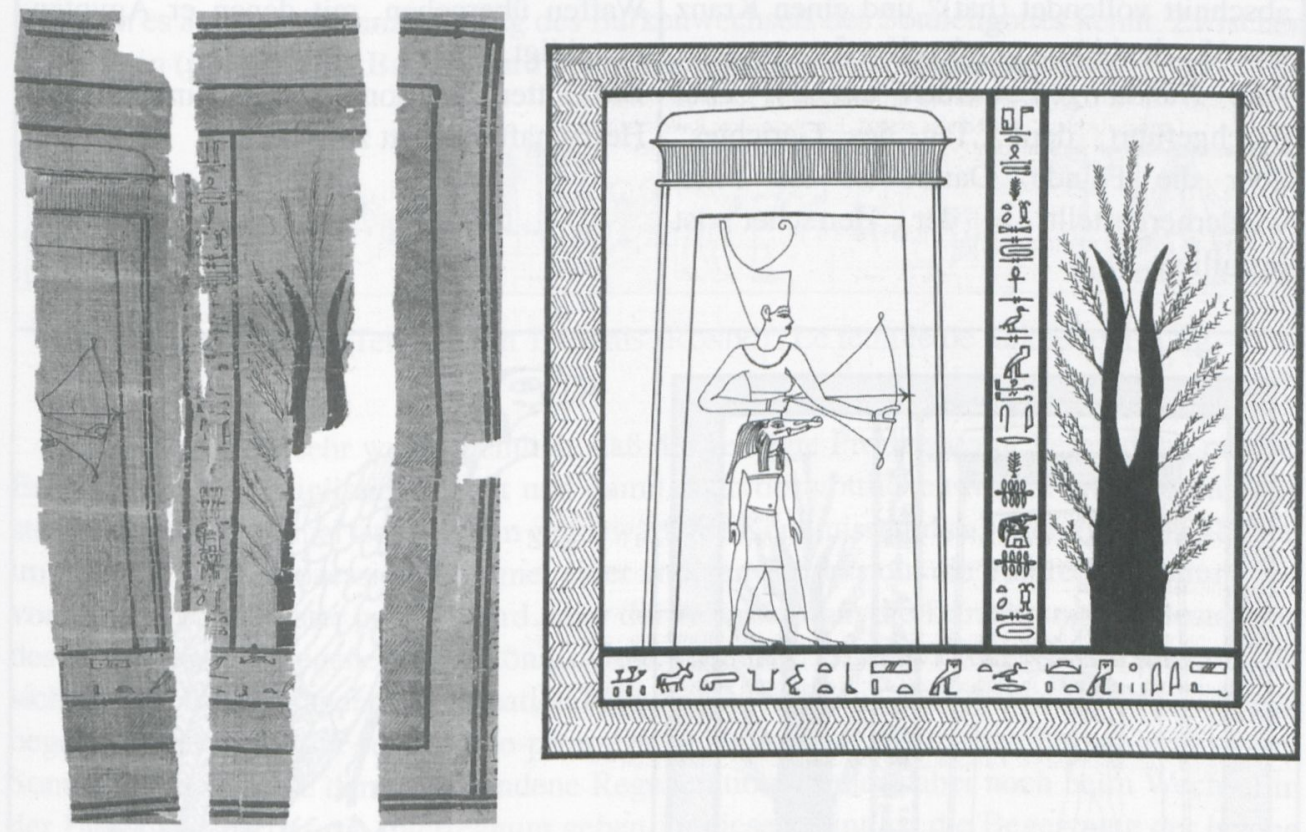

Abb. 5 „Akazie der Neith“, Original und Rekonstruktion

Wenn man nun das Geschehen im „Lebenshaus von Ra-sehet“ mit den Ritualen der „Confirmation du pouvoir royal au nouvel an“17 verbindet, so bietet sich für „Akazie der Neith“ die Rolle des ,sh $n$ smn $j w^{c} \cdot t^{\prime \prime}$ an. Der Ort wäre wie Chemmis ein unzugänglicher Platz, eine Insel, wo die Gottesmutter den Erben (jw`.w) schützt, bis er alt genug ist, um sein Erbe anzutreten. Chemmis ist dabei allerdings der Ort, der seine Rolle in der Verbindung mit den Mythen und Riten um Osiris, Isis und Horus hat. Wenn man für Isis „Mutter“ einsetzt und für Horus „Erbe“, dann müßte man im Falle des Sobek für seine Mutter, die ihn schützt „Neith“ einsetzen. Der Aufenthalt des Sobek an diesem Platz wäre damit die Wiederholung des Zeitabschnittes, in dem Sobek bis zu seiner Volljährigkeit, mit der er die Erb-Befähigung erlangt, geschützt wird.

17 J.-CL. Goyon, Confirmation du pouvoir royal au nouvel an: Brooklyn Museum papyrus 47.218.50. Textband, BdÉ 52, Le Caire 1972. Tafelband Wilbour monographs 7, 1974. 


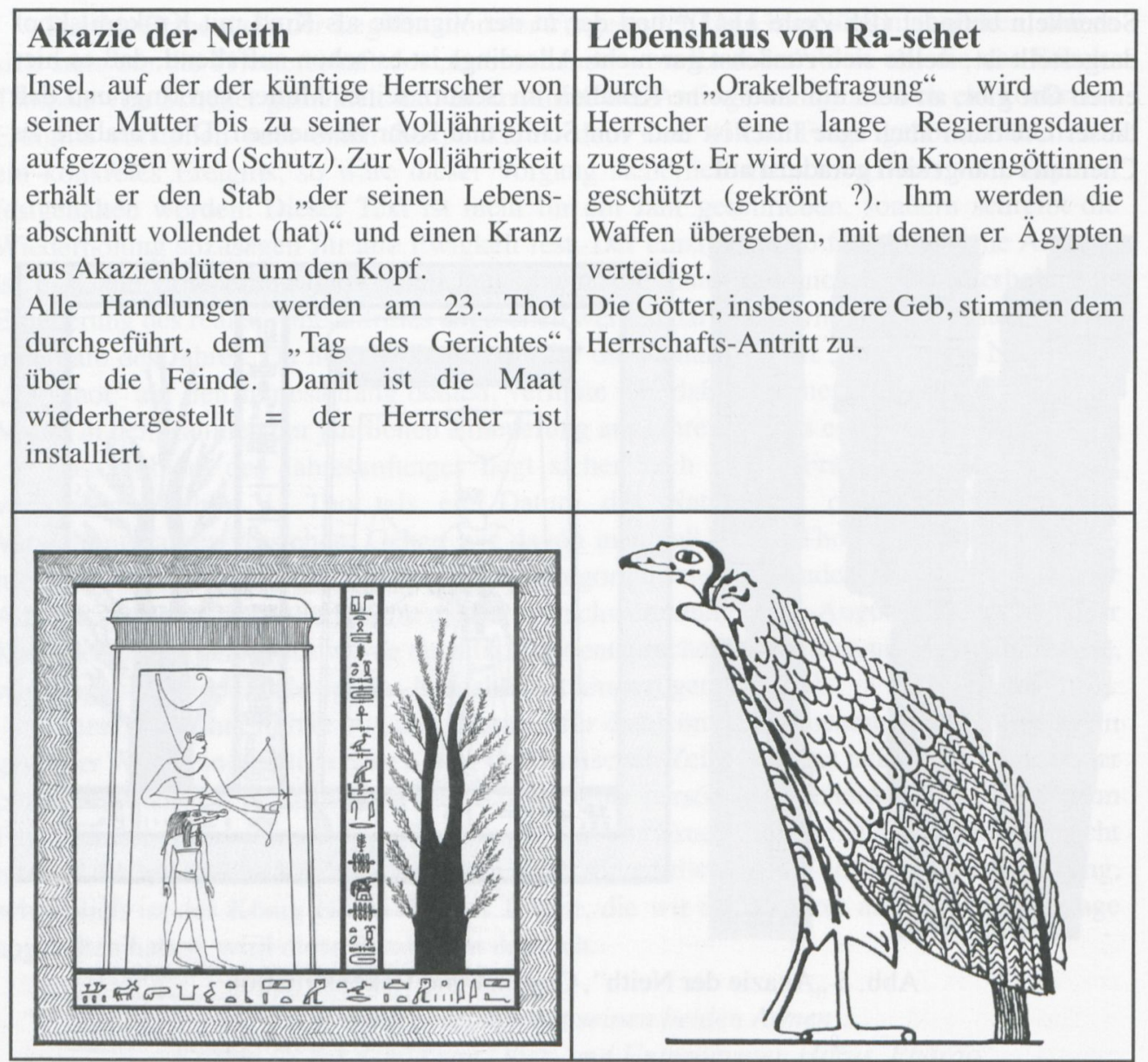

Das Ritual auf der Insel, von dem das „Buch vom Fayum“ berichtet, wird am 23. Thot vollzogen. D.h. es ist ein jährliches Ritual. Dieser Tag ist der „Tag des Gerichtes“, eine „rite de passage": Alles Feindliche, was das Kind hätte bedrohen können und wovor die Mutter ihn beschützte, wird jetzt abgeurteilt. Die Maat ist wiederhergestellt. Der Schutz der Mutter und der Insel ist nicht mehr nötig. Als Zeichen der Volljährigkeit erhält der Herangewachsene einen Kranz ${ }^{18}$ von Akazienblüten um den Kopf. Als Zeichen seiner Handlungsfähigkeit erhält er vom Priester des Sobek einen 4 Ellen langen Stab aus Akazienholz mit Löwenknauf, der den Namen hat: „der seinen Lebensabschnitt vollendet (hat) $\left(s k m-{ }^{\complement} h^{\complement}=f\right)^{\prime \prime}$. Die letzten Worte zum Ort „Akazie der Neith“ klingen wie eine Ovation: „Es sei gefestigt die Lebenszeit des Herrn der Beiden Länder (smn $h^{\ulcorner} n n b$-t3.wj)" ! Hier scheint auch eine Beziehung zum Namen des Ritualortes, $s h$ h $n$ sm $j w^{`} . t$-Kapelle für die Festigung des Erbes“ zu bestehen.

Die Fortsetzung des Rituals findet anscheinend im Lebenshaus von Ra-sehet statt. Der Herrscher hat durch die Volljährigkeit die Regierungsfähigkeit erlangt. Er ist durch seine Herkunft legitimiert. Jetzt wird er zum Lebenshaus von „Ra-sehet“ gebracht, um dort die

18 Es wird im Text nicht von einem Kranz gesprochen, sondern es heißt nur,,Die Akazie, zusammengeknüpft sind ihre Blüten an seinem Kopf ..... 
Zustimmung der Götter zu seiner Herrschaft zu erhalten. Dies geschieht, wie schon gezeigt wurde, durch Rituale und Weissagungen.

Noch eine weitere Darstellung könnte in den Komplex „Erneuerung der königlichen Herrschaft" gehören. Die Reliefdarstellungen des Tempels von Tebtynis ${ }^{19}$ zeigen eine Prozession der beiden Barken (links), die durch ihre Darstellung im 2. Papyrusabschnitt des „Buches vom Fayum“ die Konnotation der Regeneration haben. Sie sind dort so dargestellt, wie man es aus dem Zusammenhang des Barkenwechsels des Sonnengottes kennt. Zwischen den Bugen (prows) ${ }^{20}$ der Barken wird der Sonnengott aus dem Wasser geboren.

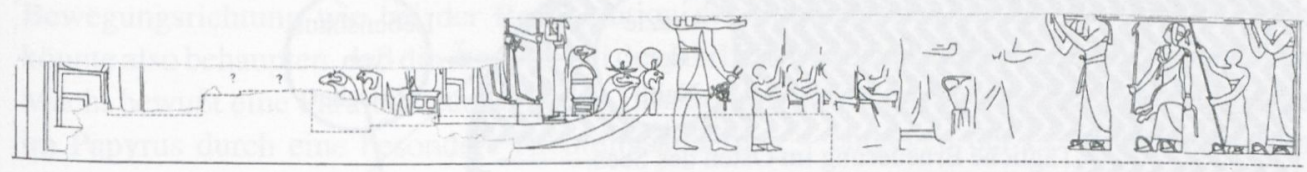

Abb. 6 Prozession im Tempel von Tebtynis (RonDot, Le temple de Soknebtynis, fig. 47)

Ich halte es für sehr wahrscheinlich, daß die gezeigte Prozession in Verbindung mit der Erneuerung der königlichen Macht und damit auch der göttlichen Macht am Jahresanfang steht, denn dieses Ritual ist nach dem gegenwärtigen Kenntnisstand das wichtigste der Tempel im Fayum. Diese Prozession begegnet einer anderen Prozession, die von rechts kommt und von einem Sempriester geführt wird. Hier dürfte es sich um die Darstellung der Bestattung des Vorgängers des regenerierten Königs/Gottes handeln. Wie das in der Realität aussah, läßt sich natürlich nicht sagen. Die Situation hat aber dadurch, daß sich die beiden Prozessionen begegnen, die Symbolik der prow-to-prow-Darstellungen, meint also den Barkenwechsel des Sonnengottes und die damit verbundene Regeneration. Weder dabei noch beim Wechsel in der Herrschaft darf es ein Interregnum geben. In diesem Sinn ist die Begegnung der beiden Prozessionen in Tebtynis zu verstehen.

Das Ostufer des Fayum-Sees ist von der Vorstellung der Erneuerung der königlichen bzw. göttlichen Herrschaft geprägt. Dabei findet die Regeneration des Sonnengottes täglich, die des Herrschers jährlich statt.

Wenn man den 7. Abschnitt des „Buches vom Fayum“, in dem die Urgötter und Nun das Fayum gründen, im Zusammenhang mit dem eben Erarbeiteten sieht, so kann man dies nur als Vorgeschichte zum Herrschaftsantritt des Sonnengottes bzw. des Herrschers im Fayum ansehen. Wahrscheinlich ist diese Erzählung von der Gründung des Fayum so notwendig für die Erneuerung der königlichen bzw. göttlichen Macht wie die Vorstellung, daß dem Regierungsantritt des ägyptischen Königs eine Zeit des Chaos vorangeht.

Die Teilung des „Buches vom Fayum“ in zwei - zusammengehörige - Handschriften, wie wir sie in der Version „Boulaq/Hood/Amherst“ haben, scheint doch weniger zufällig zu sein als bisher zu vermuten war. Beide Hälften ${ }^{21}$ stellen für sich eine Einheit dar, die über das Optische hinausgeht. In der ersten Hälfte geht es um die Herrschaft des Sonnengottes und im geringeren Maße - um die Rolle des Osiris. Es ist die eher göttliche Sphäre.

19 V. Rondot, Le temple de Soknebtynis et son dromos, Tebtynis II, Kairo 2004, 129-133, photo 94 und fig. 47.

20 Der Ausdruck „Bug“ ist besonders im Plural „Buge“ oder „Büge“ auch im Deutschen nicht jedermann verständlich. Ich erlaube mir deshalb, das englische „prow“ daneben zu setzen.

21 Das ist hier nicht im mathematischen Sinn gemeint. 
Erneuerung der königlichen / göttlichen Herrschaft

Legitimation durch

Herkunft (Schutz

durch die Mutter bis

zur Volljährigkeit)

(Chemmis-Vorstellung)

Süden

Jährliche Erneuerung

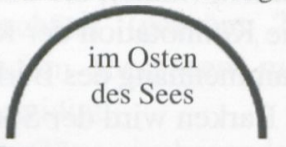

Legitimation durch göttliche Zustimmung (Schutz durch die Götter)

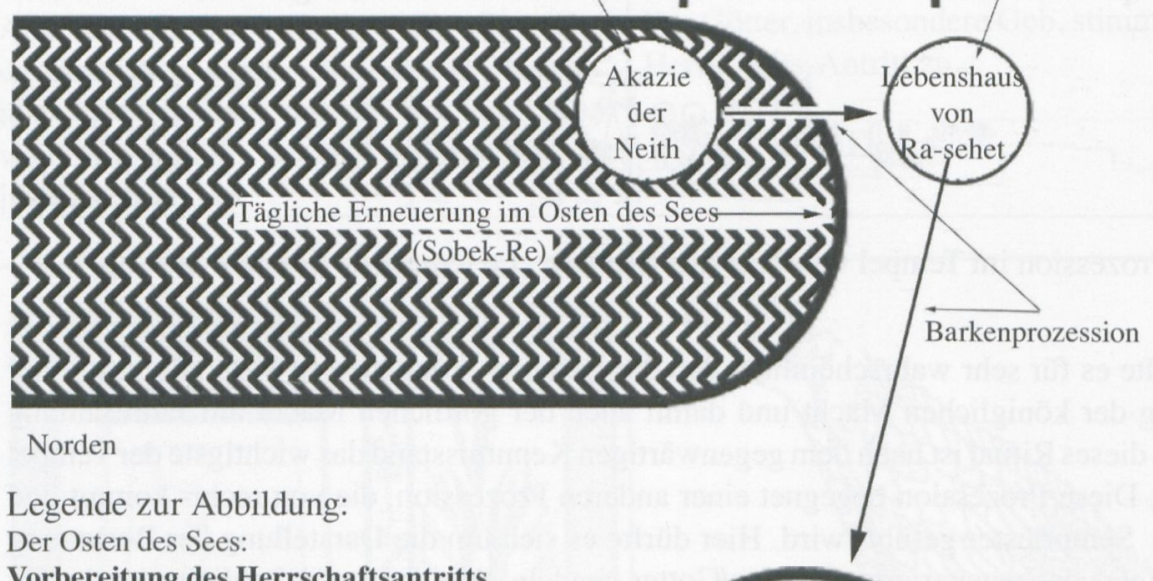

Vorbereitung des Herrschaftsantritts

1. (Sonnen)Gott : Regeneration aus dem Wasser (=Schutz der Mutter) - täglich

2. König : Kindheit im Schutz der Insel (=Schutz der Mutter) - jährlich

Herrschaftsantritt

1. (Sonnen)Gott : Wird mit Hilfe der

Urgötter von der Himmelskuh an den

Himmel gehoben.

2. König : Übernimmt mit Zustimmung der Götter die Herrschaft.

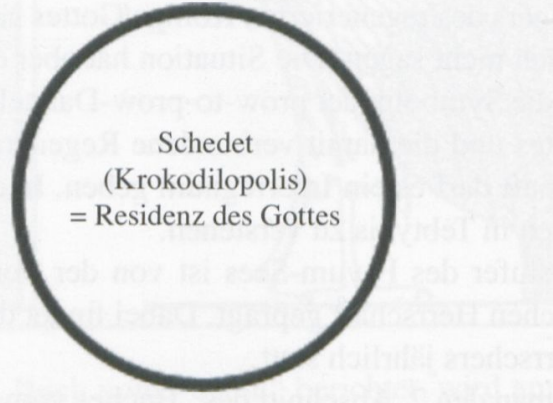

Abb. 7 Schema der Erneuerung der königlichen / göttlichen Herrschaft im Fayum

Auf ein Phänomen ist noch aufmerksam zu machen, auf den Unterschied zwischen der Leserichtung des Papyrus und dem logischen Ablauf der Handlungen, wie sie im „Buch vom Fayum" verzeichnet sind.

Die Leserichtung des „Buches vom Fayum“"22 verläuft von links nach rechts. Diese Leserichtung ist anders als die bei vergleichbaren Papyri und erklärt sich dadurch, daß der Papyrus an die vier Himmelsrichtungen angepaßt ist und dabei oben $=$ Süden, links $=$ Osten, rechts $=$ Westen und unten $=$ Norden gilt. Die Leserichtung von links nach rechts ist also die Bewegungsrichtung der Sonne von Osten nach Westen bzw. von links nach rechts. Es ist auch die Bewegungsrichtung des Überschwemmungswassers (= Osiris) vom Niltal im Osten ins Fayum im Westen. 
Wenn es nun im dritten Papyrusabschnitt inhaltlich um den Weg der Sonne von Westen nach Osten geht, ist diese Bewegungsrichtung konträr zur Leserichtung des Papyrus. Wir können natürlich nicht sagen, ob die Ägypter darin ein Problem gesehen haben. Die hieratischen Abschriften des „Buches vom Fayum“ beachten es nicht.

Auch für das Thema der Erneuerung der königlich/göttlichen Macht ist die Frage der Richtung nicht unbedingt entscheidend. Auffallend ist aber doch, daß der logische Ablauf von der Gründung des Fayum (7. Abschnitt) über „Akazie der Neith“ (6. Abschnitt), Lebenshaus von Ra-sehet (5. Abschnitt) und Schedet (4. Abschnitt) der Leserichtung entgegenläuft. Damit haben wir also bei der Erneuerung der königlich/göttlichen Macht die gleiche Bewegungsrichtung wie bei der Regeneration des Sonnengottes im See des Fayum. Man könnte also behaupten, daß die Ägypter bei der jährlichen Erneuerung der königlich/göttlichen Macht bewußt eine Parallele zur täglichen Erneuerung des Sonnengottes Re gesehen und sie im Papyrus durch eine besondere Richtungsänderung - entgegen der Leserichtung - zum Ausdruck gebracht haben.

\section{Literatur:}

H. BeinLICH, Das Buch vom Fayum: zum religiösen Eigenverständnis einer ägyptischen Landschaft. Text- und Tafel-Bd., ÄA 51, Wiesbaden 1991.

H. BeINLICH, Der Mythos in seiner Landschaft: das ägyptische „Buch vom Fayum“. Bd. 1: Die hieroglyphischen Texte. Text- und Tafel-Bd., SRaT 11, 1, Dettelbach 2013.

H. BeINlich, Der Mythos in seiner Landschaft: das ägyptische „Buch vom Fayum“, Bd. 2: Die hieratischen Texte, SRaT 11, 2, Dettelbach 2014.

H. Beinlich, The Book of the Faiyum, in: H. Beinlich/R. Schulz/A. Wieczorek (eds.), Egypt's Mysterious Book of the Faiyum, Dettelbach 2013,27-77.

H. Beinlich, Das Buch vom Fayum, in: H. Beinlich/R. Schulz/A. Wieczorek (eds.), Die Entstehung der Welt: Schöpfungsmythen aus dem Alten Ägypten nach dem Buch vom Fayum, Dettelbach 2014, 27-77.

H. BeINLICH, Wiedergeburt aus dem Wasser: Kosmologische Vorstellungen der alten Ägypter nach dem "Buch vom Fayum", in: Antike Welt 5, 2014, 17-21. 